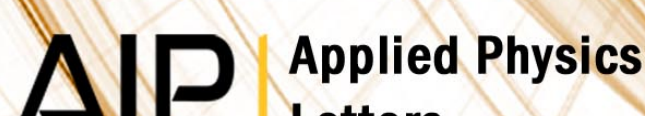 Letters
}

\section{The influence of quantum dot size on the sub-bandgap intraband photocurrent in intermediate band solar cells}

Alexander Mellor, Antonio Luque, Ignacio Tobías, and Antonio Martí

Citation: Appl. Phys. Lett. 101, 133909 (2012); doi: 10.1063/1.4755782

View online: http://dx.doi.org/10.1063/1.4755782

View Table of Contents: http://apl.aip.org/resource/1/APPLAB/v101/i13

Published by the American Institute of Physics.

\section{Related Articles}

InGaP-based InGaAs quantum dot solar cells with GaAs spacer layer fabricated using solid-source molecular beam epitaxy

Appl. Phys. Lett. 101, 133110 (2012)

An energy-harvesting scheme utilizing Ga-rich Culn(1-x)GaxSe2 quantum dots for dye-sensitized solar cells Appl. Phys. Lett. 101, 123901 (2012)

The effect of photonic bandgap materials on the Shockley-Queisser limit

J. Appl. Phys. 112, 064501 (2012)

Holographic modification of TiO2 nanostructure for enhanced charge transport in dye-sensitized solar cell

J. Appl. Phys. 112, 043110 (2012)

Relation between charge carrier density and lifetime in polymer-fullerene solar cells

J. Appl. Phys. 112, 044502 (2012)

\section{Additional information on Appl. Phys. Lett.}

Journal Homepage: http://apl.aip.org/

Journal Information: http://apl.aip.org/about/about_the_journal

Top downloads: http://apl.aip.org/features/most_downloaded

Information for Authors: http://apl.aip.org/authors

\section{ADVERTISEMENT}
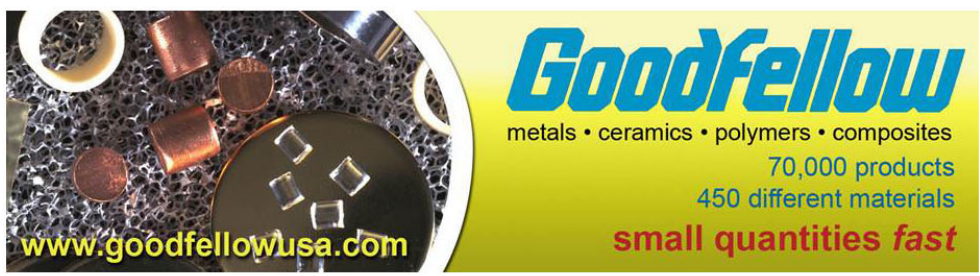


\title{
The influence of quantum dot size on the sub-bandgap intraband photocurrent in intermediate band solar cells
}

\author{
Alexander Mellor, Antonio Luque, Ignacio Tobías, and Antonio Martí \\ Instituto de Energía Solar, Universidad Politécnica de Madrid, 28040 Madrid, Spain
}

(Received 30 March 2012; accepted 13 September 2012; published online 27 September 2012)

\begin{abstract}
The effect of quantum dot (QD) size on the performance of quantum dot intermediate band solar cells is investigated. A numerical model is used to calculate the bound state energy levels and the absorption coefficient of transitions from the ground state to all other states in the conduction band. Comparing with the current state of the art, strong absorption enhancements are found for smaller quantum dots, as well as a better positioning of the energy levels, which is expected to reduce thermal carrier escape. It is concluded that reducing the quantum dot size can increase sub-bandgap photocurrent and improve voltage preservation. (C) 2012 American Institute of Physics. [http://dx.doi.org/10.1063/1.4755782]
\end{abstract}

The intermediate band solar cell (IBSC) is a high efficiency solar cell concept whose detailed balance efficiency limit has been calculated as $63 \%,{ }^{1}$ to be compared to the Schockly-Queisser limit of $41 \%^{2}$ for conventional singlebandgap solar cells (both values have been calculated assuming maximum light concentration and treating the sun as a blackbody at $6000 \mathrm{~K}$ ). The concept is based on the introduction of an IB between the valence band (VB) and conduction band (CB) of a semiconductor, thus, constituting a so-called IB material. The IB allows an increase in photocurrent via a two sub-bandgap photon absorption process in which one photon promotes an electron from the VB to the IB and another promotes an electron from the IB to the $\mathrm{CB}$, creating a single electron-hole pair. For the IBSC to exceed the efficiency of a conventional single-gap solar cell, this additional sub-bandgap photocurrent must be delivered whilst maintaining an open circuit voltage $\left(\mathrm{V}_{\mathrm{oc}}\right)$ that is limited by the overall (VB-CB) bandgap of the device and not by one of the smaller (VB-IB or IB-CB) sub-bandgaps. For this to occur, the quasi Fermi levels describing the carrier populations in each of the three bands must separate under operating conditions. ${ }^{1}$

IBSCs have been realized in which the IB is constituted by the ground state of InAs quantum dots (QDs) in a GaAs matrix. ${ }^{3-7}$ The bandgaps of this system are not optimal, but it has allowed the basic principles of IB operation to be proven. ${ }^{8,9}$ Two of the main problems experienced by these devices are that the direct optical IB-CB transition is too weak, ${ }^{10}$ and that there is strong thermal coupling between the IB and the $\mathrm{CB},{ }^{11}$ preventing the splitting of the IB and $\mathrm{CB}$ quasiFermi levels at room temperature.

A simplified spatial band diagram of the QD-IBSC is shown in Figure 1. The CB offset between the QD and matrix materials presents a potential well. The confining potential of the well leads to a set of discrete states that are highly localized within the QD. These may have a lower energy than the confining potential, in which case they are termed bound states (BSs - shown as blue lines), or may have a higher energy than the confining potential, in which case they are termed virtual bound states (VBSs - shown as red lines). In addition, there is a continuum of extended states
(ESs) within the energy range of the matrix material conduction band. These are schematically represented as a green area. The CB of the IBSC is defined to constitute all states with greater energy than the confining potential. The IB is defined as the QD ground state, labelled $(1,1,1)$ in the figure.

In current InAs/GaAs QD-IBSCs, there are multiple BSs within the forbidden band of the matrix material. It has recently been shown that the gas of thermal photons, present in the solar cell at ambient temperatures, can cause thermal escape by optical excitation of electrons from the IB to the $\mathrm{CB}$ using the multiple excited states within the forbidden band as a ladder. ${ }^{12}$ This process dominates over pumping by photons incident from the sun in the absence of concentration. Hence, these excited states should be removed from the forbidden band in order to supress thermal coupling between the IB and the $\mathrm{CB}$. What is more, the strength of the direct optical IB-CB transition must be increased so that electrons pumped to the IB from the VB can be efficiently pumped to the $\mathrm{CB}$ under illumination. In this letter, we argue, on the basis of quantum calculation of intersubband transitions, that both of these objectives can be achieved by modifying the QD dimensions.

We take as a benchmark the prototype QD-IBSC system that was reported on in Refs. 11 and 13, in which it was named SB and S3, respectively. This system consisted of a 30-layer stack of $\operatorname{In}(\mathrm{Ga}) \mathrm{As} / \mathrm{GaAs}$ QDs grown on a GaAs substrate. The QDs had the shape of truncated quadrangular pyramids. These are modelled as parallelepipeds in this work. The measured QD dimensions were $16 \times 16 \times 6 \mathrm{~nm}^{3}$ in the $\mathrm{x}, \mathrm{y}$, and $\mathrm{z}$ directions, respectively, where the $\mathrm{xy}$ plane is perpendicular to the growth direction. The fractional coverage of the $x y$ plane by QDs (QD area / total area) was $F=0.1$ and the number of QD layers per unit length in the $z$ direction was $N_{l}=125000 \mathrm{~cm}^{-1}$. This QD-IBSC is hereafter referred to as the benchmark prototype.

The benchmark prototype was modelled in Refs.12 and 14-16 by finding an approximate solution to the single-band effective mass equation (which is the outcome of the one dimensional $\mathrm{k} \cdot \mathrm{p} \mathrm{model}{ }^{17}$ ) by a separation of the $x, y$, and $z$ variables. This model is simpler than others that appear in the literature. ${ }^{5,18}$ Its use allows us to perform parameter 

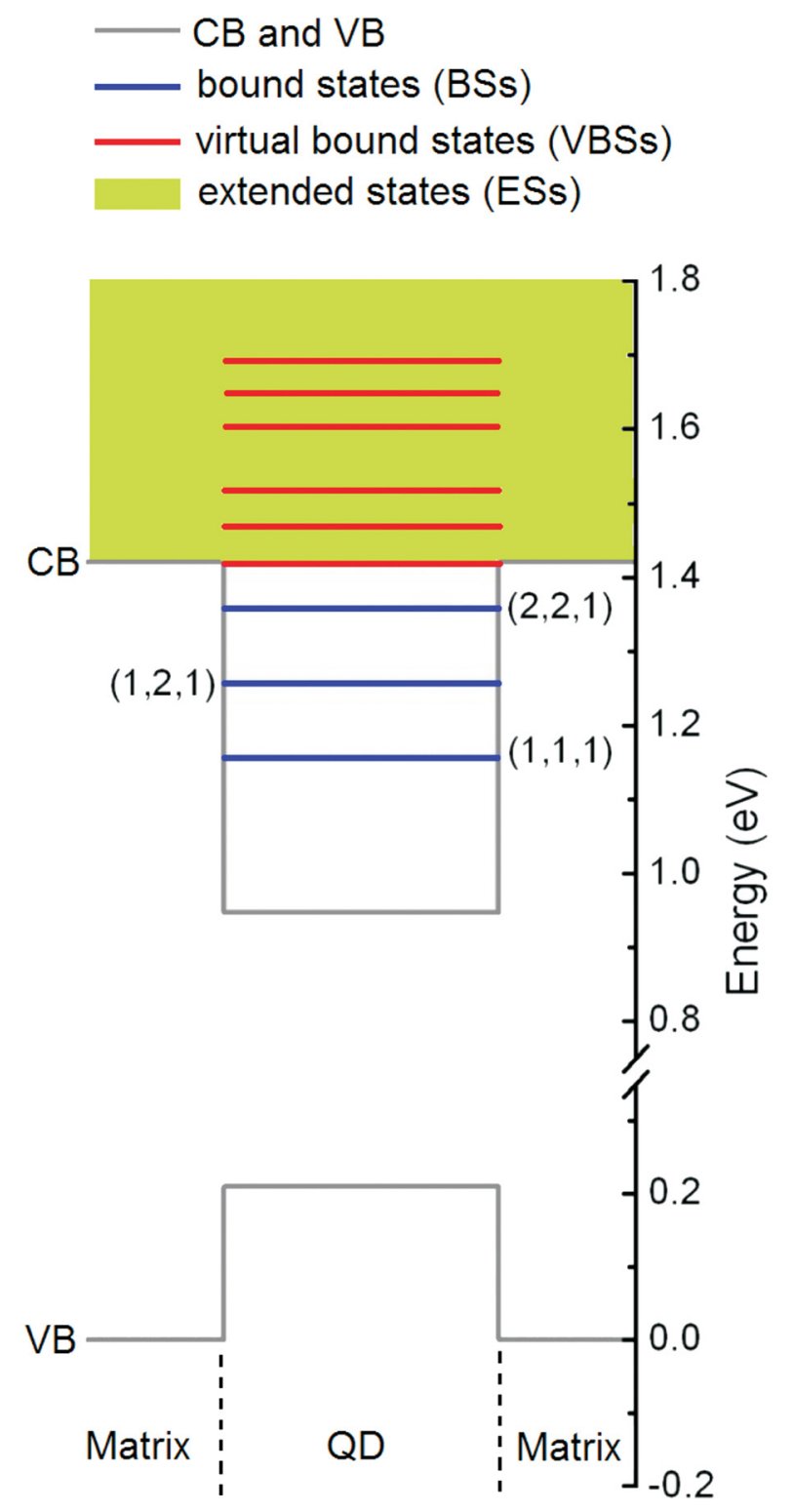

FIG. 1. Simplified band diagram of the QD-IB material. The CB and VB are shown in grey, the BSs in blue, the VBSs in red, and the continuum of ESs in green. BSs are labelled with their quantum numbers as defined in Ref. 15 . In this figure, the energy origin is at the matrix VB edge.

sweeps without the need for large computational capacity. The spin-orbit coupling and lattice strain are not accounted for explicitly in the model; however, they are taken account of implicitly in the experimental effective mass (0.0294) and the CB offset $(0.473 \mathrm{eV}) .{ }^{19,20}$ A four-band extension of the model has been used to accurately predict quantum efficiencies (QEs) of the benchmark prototype. ${ }^{15}$ Justification of the use of the single-band model for intersubband transitions can be found in the same reference. In this work, the model has been applied to QDs of different dimensions. All other parameters are maintained the same as the benchmark prototype. Fixing $F$ and $N_{l}$ at the above stated values implies the volume density of QDs is conserved. The CB offset would be expected to change on changing the QD size; however, this effect is not considered in this work.

Figure 2 shows the energy levels (measured from the $\mathrm{CB}$ edge of the matrix material) of all the BSs that exist within the forbidden band as a function of the lateral dot
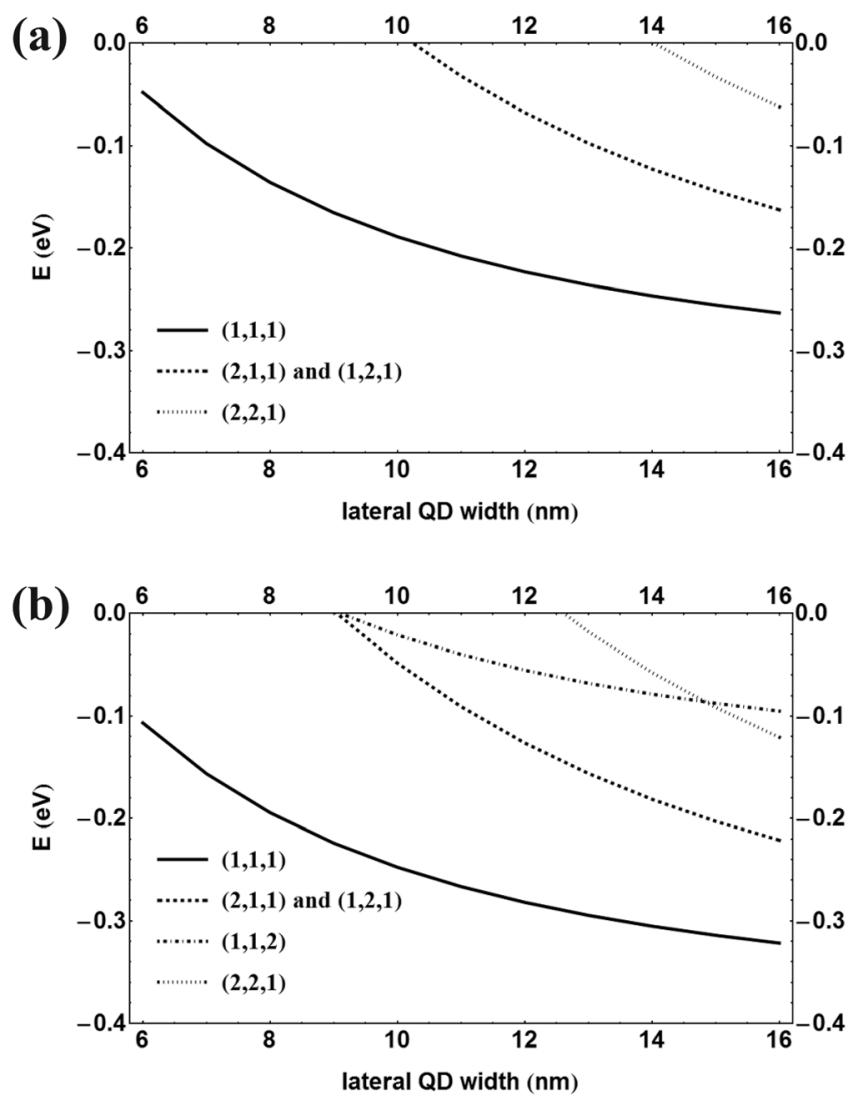

FIG. 2. BS energy levels as a function of the QD width for QDs of height $6 \mathrm{~nm}$ (a) and $9 \mathrm{~nm}$ (b). States are labelled by their quantum numbers as defined in Ref. 15. The energy origin is at the matrix CB edge.

width. Their quantum numbers following the nomenclature in Ref. 16 are given in the legend. Figure 2(a) is for a fixed QD height of $6 \mathrm{~nm}$ (the benchmark height) and Figure 2(b) is for a fixed height of $9 \mathrm{~nm}$.

It should be noted that, for a QD-IB material formed in a GaAs matrix (bandgap $1.42 \mathrm{eV}$ ), the optimum energy position of the ground state is around $0.5 \mathrm{eV}$ from the matrix $\mathrm{CB}$ edge. ${ }^{1}$ Hence it is desirable to maintain a ground state energy that is as low as possible whilst removing excited states from the forbidden band. In Figure 2(a), this is achieved for QD dimensions of $10 \times 10 \times 6 \mathrm{~nm}^{3}$. In Figure 2(b), this is achieved for QD dimensions of $9 \times 9 \times 9 \mathrm{~nm}^{3}$. The energy position of the ground state in each case is $0.19 \mathrm{eV}$ and $0.24 \mathrm{eV}$ below the matrix $\mathrm{CB}$ edge, respectively. We observe that the $9 \times 9 \times 9 \mathrm{~nm}^{3}$ cubic QD represents the optimum with respect to energy levels since the $(2,1,1),(1,2,1)$, and $(1,1,2)$ states have degenerated and coincide with the matrix CB edge; hence, no dimension can be changed without either raising the energy of the ground state or introducing one of the above mentioned levels into the forbidden band. This agrees with a similar result obtained in Ref. 20 where a spherical QD in a similar QD-IBSC system was found to have an optimum radius of $4.09 \mathrm{~nm}$ (diameter $8.18 \mathrm{~nm}$ ) with regard to energy levels. Nonetheless, we consider the $10 \times 10 \times 6 \mathrm{~nm}^{3}$ to be interesting, given the difficulty in producing QDs with low aspect ratios. It should be noted that in Ref. 14 a perturbation method was used to correct errors in the ground state energy levels resulting from the use of the separation-of-variables method. The energy corrections were $-2.78 \times 10^{-8} \mathrm{eV}$ for the benchmark QD and $-0.021 \mathrm{eV}$ for 


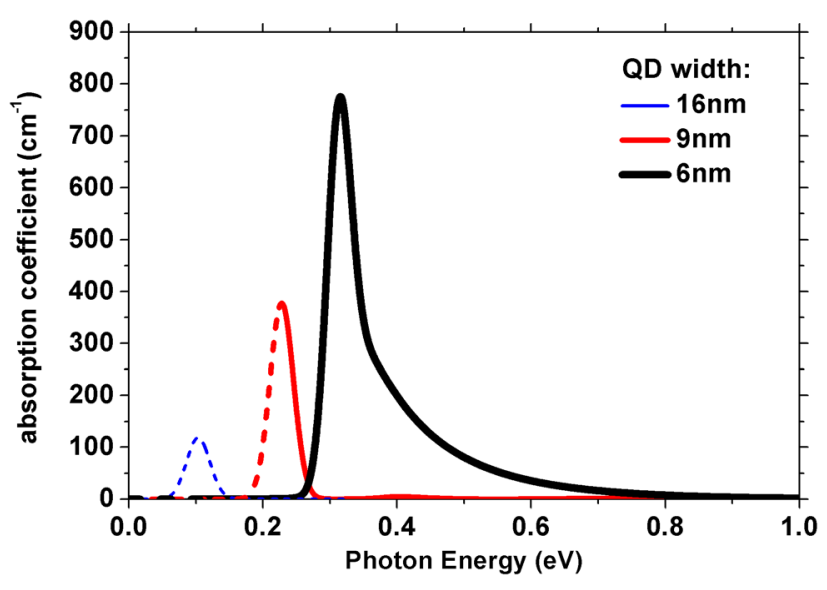

FIG. 3. Absorption coefficients for transitions from the ground BS (IB level) under unpolarised normally incident illumination. Different curves are for QDs with different widths, as specified in the legend. The QD height is $6 \mathrm{~nm}$. The ground BS is assumed half full and all other states are assumed empty. Solid parts of the curves represent transitions whose final state is within the matrix $\mathrm{CB}$ and dashed parts represent transitions whose final states are within the forbidden band.

a cubic QD. Changes of this scale in the energy levels shown in Figure 2 would affect the optimum QD width by less than $0.5 \mathrm{~nm}$.

We now study the effect of the QD dimensions on the absorption coefficient for transitions originating from the ground state under unpolarised illumination incident in the $z$ direction. This is calculated by summing the contributions of transitions to all other states. It is assumed that the ground state has a 0.5 probability of occupation by an electron, due to appropriate doping, and that all other states have 0 probability of occupation. The QD height is fixed at $6 \mathrm{~nm}$ and the absorption coefficient is calculated for a range of widths. These are plotted in Figure 3. The QD width corresponding to each curve is shown in the figure legend. For a transition to contribute to the external current, its final state must be within the energy range of the matrix $\mathrm{CB}$. The solid parts of the curves in Figure 3 represent transitions that fulfil this condition; the dashed parts represent transitions that do not.

For each QD width, a single peak can be observed, which corresponds to the transition from the ground state to the doubly degenerate $(2,1,1) /(1,2,1)$ state. On decreasing the QD width, the absorption by this transition increases significantly due to the increased number density of QDs. Also, its final state moves into the matrix $\mathrm{CB}$ energy range (as can also be seen in Figure 2(b)). For the $6 \mathrm{~nm}$ wide QD, a tail extending into higher energies can also be seen. This corresponds to transitions to states that are extended in one dimension and bound in the other two. These transitions drop off quickly at higher energies and are negligibly weak for larger QDs, which agree with previous numerical ${ }^{21,22}$ and experimental $^{23}$ studies. In all cases, the absorption coefficient is negligible in the energy range for which VB-IB transitions occur $(\hbar \omega>0.9 \mathrm{eV})$. This is desirable for IBSC operation, since photons with sufficient energy for VB-IB transitions are not wasted in contributing to the lower energy IB-CB transition. It should be noted that, under the separation of variables approximation, the curves presented in Figure 3 have the same shape for any QD height, although the energy gap between the ground state and the matrix CB differs.
Experimental $^{11}$ and numerical ${ }^{15}$ studies have shown VB-IB photocurrents of 0.266 and $0.208 \mathrm{~mA} \mathrm{~cm}^{-2}$, respectively, for the benchmark prototype (the quoted experimental value was calculated in Ref. 15 from internal quantum efficiency measurements presented in Ref. 11). To see if the absorption coefficients presented in Figure 3 are strong enough to deliver this photocurrent from the IB to the $\mathrm{CB}$, an absorbed photocurrent density for the IB-CB transition has been calculated using the formula

$$
J_{p h, I B-C B}=q_{e} \int_{E_{C B}-E_{1,1,1}}^{E_{1,1,1}-E_{V B}} \Phi_{B B}\left(1-e^{-\alpha(E) W}\right) d E
$$

where $q_{e}$ is the elementary charge, $W$ is the width of the IB material $(2.4 \mu \mathrm{m})$ and $\Phi_{B B}$ is the spectral photon flux of the sun, which is modelled as a black body at $5762 \mathrm{~K}$, incident on the cell at $1 \times$ concentration. The absorption coefficient $\alpha(E)$ is calculated assuming the same Fermi occupations as before. The actual Fermi occupations will depend on the relative strengths of the VB-IB and IB-CB transitions; however, the assumptions used here serve for a comparative analysis. It should also be noted that the IB Fermi level can to some extent be controlled by doping, as long as the VB-IB and IBCB transitions are of similar strength.

In Figure $4, J_{p h, I B-C B}$ is plotted as a function of the lateral QD width for QDs with a fixed height of $6 \mathrm{~nm}$ (dashed curve) and $9 \mathrm{~nm}$ (solid curve). For a QD width of $16 \mathrm{~nm}$, the calculated $J_{p h, I B-C B}$ is $0.005 \mathrm{~mA} \mathrm{~cm}^{-2}$, to be compared with the measured $0.266 \mathrm{~mA} \mathrm{~cm}^{-2}$ for the VB-IB transition. This is in agreement with the hypothesis that in the benchmark prototype the VB-IB photocurrent was extracted from the solar cell due to thermal escape and not pumping by photons incident from the illuminating source. Arrows in Figure 4 denote the $10 \times 10 \times 6 \mathrm{~nm}^{3}$ and $9 \times 9 \times 9 \mathrm{~nm}^{3}$ QDs, which are identified as providing optimum energy levels previously in this letter. Steps in the $J_{p h, I B-C B}$ occur at these parameter sets due to the $(2,1,1) /(1,2,1)$ states and the corresponding absorption peak entering into the matrix $\mathrm{CB}$ energy range. The breadth of these steps depends on the standard deviation

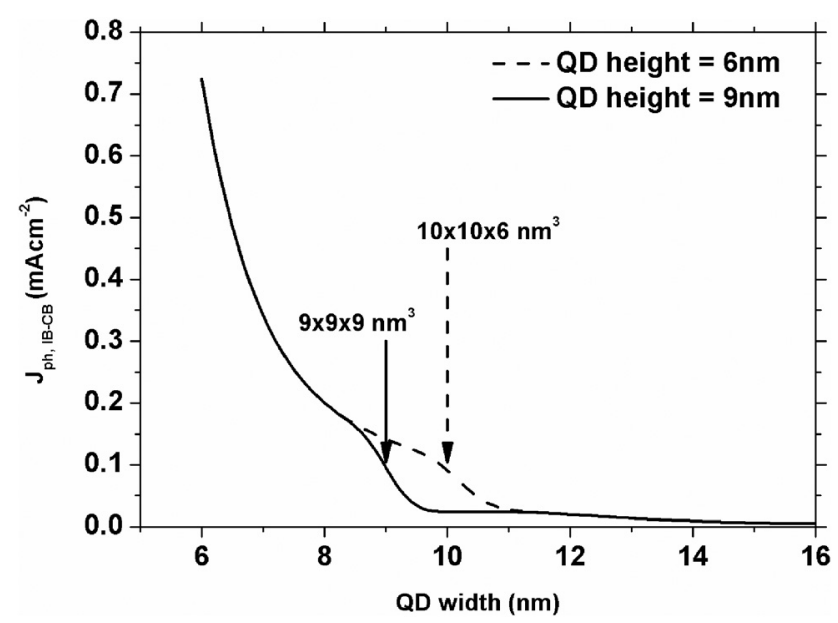

FIG. 4. The absorbed photocurrent density for the IB-CB transition $\left(J_{p h, I B-C B}\right)$ as a function of the QD width. Calculations are made for QDs of height $6 \mathrm{~nm}$ (dashed curve) and of height $9 \mathrm{~nm}$ (solid curve). 
TABLE I. The calculated $J_{p h, I B-C B}$ and the ground state energy $\mathrm{E}_{(1,1,1)}$ for three sets of QD dimensions. The ground state energy is measured from the matrix $\mathrm{CB}$ edge.

\begin{tabular}{lccc}
\hline \hline QD dimensions & $16 \times 16 \times 6 \mathrm{~nm}^{3}$ & $10 \times 10 \times 6 \mathrm{~nm}^{3}$ & $9 \times 9 \times 9 \mathrm{~nm}^{3}$ \\
\hline$J_{p h, I B-C B}\left(\mathrm{~mA} \mathrm{~cm}^{-2}\right)$ & 0.005 & 0.091 & 0.095 \\
$\mathrm{E}_{(1,1,1)}(\mathrm{eV})$ & -0.26 & -0.19 & -0.24 \\
\hline \hline
\end{tabular}

of the Gaussian function used to describe the broadening due to variations in the QD dimensions across the array. In these calculations, a standard deviation of $25 \mathrm{meV}$ was used. Just to the left of the steps, a $J_{p h, I B-C B}$ of around $0.15 \mathrm{~mA} \mathrm{~cm}^{-2}$ is predicted. This is comparable to the VB-IB photocurrents measured in quantum efficiency experiments. The $J_{p h, I B-C B}$ continues to increase on further decreasing the QD width. However, this comes at the cost of increasing the ground state energy, as has been discussed with regard to Figure 2. Nonetheless, the benefit of decreasing the QD width to $8-10 \mathrm{~nm}$ is clear.

Our results are summarized in Table I, which presents the calculated $J_{p h, I B-C B}$ and the energy level of the ground state measured from the matrix $\mathrm{CB}$ edge. Data are presented for the benchmark $16 \times 16 \times 6 \mathrm{~nm}^{3} \mathrm{QD}$, as well as for the $10 \times 10 \times 6 \mathrm{~nm}^{3}$ and $9 \times 9 \times 9 \mathrm{~nm}^{3}$ parameter sets, for which no excited states exist in the forbidden band. The ground state of the $9 \times 9 \times 9 \mathrm{~nm}^{3}$ is preferable to that of the $10 \times 10 \times 6 \mathrm{~nm}^{3}$, although the $10 \times 10 \times 6 \mathrm{~nm}^{3}$ QD may be easier to realise in practice. The $J_{p h, I B-C B}$ for both the $10 \times 10 \times 6 \mathrm{~nm}^{3}$ and $9 \times 9 \times 9 \mathrm{~nm}^{3}$ QDs are around $0.09 \mathrm{~mA}$ $\mathrm{cm}^{-2}$ : a nearly 20 -fold increase compared to the benchmark. These values are comparable to the VB-IB photocurrents in the benchmark prototype measured in quantum efficiency experiments. We conclude that reducing the QD width in QD-IBSCs could reduce thermal escape whilst allowing carriers generated from the VB to the IB to be removed to the $\mathrm{CB}$ by absorption of photons incident from the sun at $1 \times$ concentration. Hence this is proposed as a strategy for realising simultaneous photocurrent enhancement and $\mathrm{V}_{\mathrm{oc}}$ preservation at room temperature.

This work has been supported by the Spanish Ministry of Science and Innovation through the project NANOGEFES (Grant No. ENE2009-14481-C02-01) and the EC through the project NGCPV (Grant No. 283798). Alexander Mellor gratefully acknowledges the Comunidad de Madrid for financial support through the scholarship Personal Investigador de Apoyo.

${ }^{1}$ A. Luque and A. Martí, Phys. Rev. Lett. 78, 5014-5017 (1997).

${ }^{2}$ W. Shockley and H. J. Queisser, J. Appl. Phys. 32, 510-519 (1961).

${ }^{3}$ A. Luque, A. Marti, C. Stanley, N. Lopez, L. Cuadra, D. Zhou, J. L. Pearson, and A. McKee, J. Appl. Phys. 96, 903-909 (2004).

${ }^{4}$ S. M. Hubbard, C. D. Cress, C. G. Bailey, R. P. Raffaelle, S. G. Bailey, and D. M. Wilt, Appl. Phys. Lett. 92, 123512 (2008).

${ }^{5}$ V. Popescu, G. Bester, M. C. Hanna, A. G. Norman, and A. Zunger, Phys. Rev. B 78, 205321 (2008).

${ }^{6}$ S. Blokhin, A. Sakharov, A. Nadtochy, A. Pauysov, M. Maximov, N. Ledentsov, A. Kovsh, S. Mikhrin, V. Lantratov, S. Mintairov, N. Kaluzhniy, and M. Shvarts, Semiconductors 43, 514-518 (2009).

${ }^{7}$ R. Oshima, A. Takata, and Y. Okada, Appl. Phys. Lett. 93, 083111 (2008).

${ }^{8}$ A. Luque, A. Marti, N. Lopez, E. Antolin, E. Canovas, C. Stanley, C. Farmer, L. J. Caballero, L. Cuadra, and J. L. Balenzategui, Appl. Phys. Lett. 87, 083505 (2005).

${ }^{9}$ A. Martí, E. Antolín, C. R. Stanley, C. D. Farmer, N. López, P. Díaz, E. Cánovas, P. G. Linares, and A. Luque, Phys. Rev. Lett. 97, 247701 (2006).

${ }^{10}$ A. Martí, C. R. Stanley, and A. Luque, in Nanostructured Materials for Solar Energy Conversion, edited by T. Soga (Elsevier Science, Amsterdam, 2006).

${ }^{11}$ E. Antolin, A. Marti, C. D. Farmer, P. G. Linares, E. Hernandez, A. M. Sanchez, T. Ben, S. I. Molina, C. R. Stanley, and A. Luque, J. Appl. Phys. 108, 064513 (2010).

${ }^{12}$ A. Luque, A. Marti, E. Antolin, P. G. Linares, I. Tobias, and I. Ramiro, AIP Adv. 1, 022125 (2011).

${ }^{13}$ E. Antolin, A. Marti, P. G. Linares, I. Ramiro, E. Hernandez, C. D. Farmer, C. R. Stanley, and A. Luque, in 35th IEEE Photovoltaic Specialists Conference (PVSC) (IEEE, 2010), pp. 000065-000070.

${ }^{14}$ A. Luque, A. Marti, E. Antolin, and P. Garcia-Linares, Sol. Energy Mater. Sol. Cells 94, 2032-2035 (2010).

${ }^{15}$ A. Luque, A. Martí, E. Antolín, P. G. Linares, I. Tobías, I. Ramiro, and E. Hernandez, Sol. Energy Mater. Sol. Cells 95, 2095-2101 (2011).

${ }^{16}$ A. Luque, A. Martí, A. Mellor, D. Fuertes Marrón, I. Tobías, and E. Antolín, "Absorption coefficient for the intraband transitions in quantum dot materials," Prog. Photovoltaics (in press).

${ }^{17}$ S. Datta, Quantum Phenomena (Addison-Wesley, Reading, Massachusetts, 1989), Vol. VII.

${ }^{18}$ S. Tomic, T. S. Jones, and N. M. Harrison, Appl. Phys. Lett. 93, 263105 (2008).

${ }^{19}$ P. G. Linares, C. D. Farmer, E. Antolín, S. Chakrabarti, A. M. Sánchez, T. Ben, S. I. Molina, C. R. Stanley, A. Martí, and A. Luque, Energy Procedia 2, 133-141 (2010).

${ }^{20}$ P. G. Linares, A. Marti, E. Antolin, and A. Luque, J. Appl. Phys. 109, 014313 (2011).

${ }^{21}$ R. Buczko and F. Bassani, Phys. Rev. B 54, 2667-2674 (1996).

${ }^{22}$ S. Tomic, Phys. Rev. B 82, 195321 (2010).

${ }^{23}$ J. Phillips, K. Kamath, X. Zhou, N. Chervela, and P. Bhattacharya, J. Vac. Sci. Technol. B 16, 1343 (1998). 